\title{
Antimicrobial Susceptibilities of Clinical Acinetobacter baumannii Isolates With Different Genotypes
}

\author{
Hatice Uludag Altun ${ }^{1, *}$; Server Yagci ${ }^{1}$; Cemal Bulut ${ }^{1}$; Hunkar Sahin ${ }^{2}$; Sami Kinikli ${ }^{1}$; Ali \\ Kudret Adiloglu ${ }^{2}$; Ali Pekcan Demiroz ${ }^{1}$ \\ ${ }^{1}$ Department of Infectious Diseases and Clinical Microbiology Clinic, Ankara Training and Research Hospital, Ankara, Turkey \\ ${ }^{2}$ Department of Medical Microbiology, Ankara Training and Research Hospital, Ankara, Turkey \\ ${ }^{*}$ Corresponding author: Hatice Uludag Altun, Department of Infectious Diseases and Clinical Microbiology Clinic, Ankara Training and Research Hospital, P.O.Box: 06340, Ankara, \\ Turkey. Tel:+31-25953000, Fax:+31-23631218, E-mail: haticeuludag80@yahoo.com
}

Received: July 04, 2013; Revised: September 1, 2013; Accepted: October 14, 2013

Background:The treatment of Acinetobacterbaumannii infections is difficult. Carbapenems, sulbactam, and colistin are the most effective antibiotics.

Objectives: The aim of this study was to evaluate the susceptibilities of genotypically different A. baumannii isolates to sulbactam, amikacin, netilmicin, meropenem, tigecycline and colistin.

Patients and Methods: Isolates from various clinical samples of patients with hospital-acquired infections that were identified by the VITEK 2 Compact system in our hospital's microbiology laboratory between January 2010 and March 2012 were included in the study. To determine genetic relatedness of the isolates, the rep-PCR method was used. The broth microdilution method was used for amikacin, netilmicin, meropenem and colistin, while E-test was used for sulbactam and tigecycline.

Results: Among the 300 isolates, 30 were found to be genotypically different and were evaluated in terms of their antimicrobial susceptibilities. All isolates were susceptible to colistin. The susceptibility rates were $66.6 \%, 50 \%, 36.6 \%, 30 \%$, and $10 \%$ for netilmicin, tigecycline, sulbactam, amikacin, and meropenem, respectively. For carbapenem resistant isolates, the susceptibility rates were $66.6 \%$, $51.8 \%, 33.3 \%$, and $25.9 \%$ for netilmicin, tigecycline, sulbactam, and amikacin, respectively. The sulbactam minimum inhibitory concentration (MIC) 50 and MIC 90 were $8 \mu \mathrm{g} / \mathrm{mL}$ and $12 \mu \mathrm{g} / \mathrm{mL}$, respectively.

Conclusions: In this study, it was concluded that determining the cut-off value for MIC breakpoints for sulbactam alone

has a critical impact on the susceptibility results.

Keywords:Acinetobacter baumannii; Sulbactam; PCR

\section{Background}

Nosocomial infections cause prolonged duration of hospitalization and increased treatment costs with high morbidity and mortality. Acinetobacter species have become the most common etiology of hospital acquired infections due to the present frequent use of wide spectrum antibiotics (1). Acinetobacter species cause pneumonia, skin and wound infections, bacteremia, and urinary tract infections while they have shown resistance to various antibiotics (2). Infections due to resistant Acinetobacter baumannii isolates cause difficulties in treatment. Carbapenems, sulbactam and colistin seem to be the most effective antibiotics for treatment (3).

Sulbactam shows high efficacy against Acinetobacter species in vitro and in vivo (4-6). Previously in Turkey, sulbactam could not be found alone; therefore sefoperazone and sulbactam combination was widely used for the treatment of multi-resistant Acinetobacter species. Today, however, commercially available sulbactam antibiotics are being used thus, the determination of the minimum inhibitory concentration (MIC) of sulbactam is necessary.

\section{Objectives}

The aim of this study was to determine the susceptibility rate of genotypically different $A$. baumannii species, isolated from various clinical samples of patients with hospital acquired infections, to sulbactam, amikacin, netilmicin, meropenem, tigecycline and colistin.

\section{Patients and Methods}

\subsection{Bacterial Isolates}

The isolates are defined as the agent of hospital acquired infections, if they emerged after 72 hours of hospitalization. All isolates were obtained from patients at intensive care units. In total, 300 A. baumannii were isolated from various clinical samples and detected by the VITEK 2 Compact system (BioMerieux, France) at the microbiology laboratory of our hospital between January 2010 and March 2012. Thirty genotypically different isolates were included in this study. The isolates were stored at $-80^{\circ} \mathrm{C}$ in brain heart infusion broth including $10 \%$ glycerol un-

Copyright ( 2014 ,Ahvaz Jundishapur University of Medical Sciences; Published by Kowsar. This is an open-access article distributed under the terms of the Creative Commons Attribution-NonCommercial 4.0 International License (http://creativecommons.org/licenses/by-nc/4.0/) which permits copy and redistribute the material just in noncommercial usages, provided the original work is properly cited. 
til susceptibility tests and genotypic evaluations were completed. According to the instructions of the manufacturer, these isolates were subjected to PCR analysis using the appropriate DiversiLab DNA Fingerprinting Kit (BioMerieux, France). Internal and external quality control strains were included in each test. An analysis of the bands was completed using the DiversiLab software 3.3 Gel images, while percentage similarity rates and dendrogram reports were formed for each DNA sample. The samples were classified as different (similarity $<95 \%$ and $>2$ band difference), similar (similarity in between 95-97\%), and indistinguishable (similarity $>97 \%, 1-2$ band difference) (7).

\subsection{Antimicrobial Susceptibility}

The susceptibilities of genotypically different isolates ( similarity $<95 \%$ and $>2$ band difference), to sulbactam, amikacin, netilmicin, meropenem, tigecycline and colistin were tested. Antibiotic susceptibilities were studied using the E-test method for sulbactam and tigecycline (BioMerieux, France), while the bouillon broth microdilution method was used for meropenem (Tumekip, Turkey), colistin, amikacin and netilmicin (Sigma Aldrich, USA). For the E-test method, bacterial suspensions with turbidity equivalent to 0.5 McFarland standard were prepared and spread onto Mueller Hinton Agar (MHA) medium (Himedia, Mumbai, India), followed by the addition of E-test strips. After the plates were incubated at $37^{\circ} \mathrm{C}$ for 24 hours, the E-test MIC value was read as the concentration at which the growth on the plate intersected the E-test strip. According to the Food and Drug Administration (FDA) criteria; if the E-test MIC value of tigecycline was $\geq 8 \mu \mathrm{g} / \mathrm{mL}$, it should be accepted as resistant, if the value was $4-6 \mu \mathrm{g} / \mathrm{mL}$, it should be accepted as intermediate, and if the value was $\leq 2 \mu \mathrm{g} / \mathrm{mL}$ it should be accepted as susceptible (8).

The cut-off value for sulbactam alone was not defined for A. baumannii isolates in the Clinical and Laboratory Standards Institute (CLSI) document. Therefore, we determined the cut-off value of sulbactam considering the values of ampicillin/sulbactam for A. baumannii isolates in the CLSI document. Accordingly, if the value was $\leq 4 \mu \mathrm{g} /$ $\mathrm{mL}$, it was accepted as susceptible, if the value was $8 \mu \mathrm{g} /$ $\mathrm{mL}$ it was accepted as intermediate and if the value was $\geq 16 \mu \mathrm{g} / \mathrm{mL}$ it was accepted as resistant. For the bouillon broth micro dilution method, cation-adjusted MuellerHinton Broth (BBL, Becton Dickinson, USA) was used according to CLSI recommendations (9). Serial dilutions of each of the four antibiotics (32-0.062 $\mu \mathrm{g} / \mathrm{mL})$ were prepared. A. baumannii isolates were suspended to 0.5 McFarland turbidity and added to micro dilution plates with 96 wells. Microplates were incubated at $35^{\circ} \mathrm{C}$ for 20-24 hours. Escherichia coli (ATCC 25922) and Pseudomonas aeruginosa (ATCC 27853) strains were used as quality control strains for all MIC determinations. The lowest antimicrobial drug concentration at which there was no growth was considered as the minimum inhibitory concentration (MIC). The results were interpreted according to CLSI criteria (9).

\section{Results}

Among the 300 A. baumannii isolates the susceptibilities of 30 isolates were determined as genotypically different (Figure 1). Twenty-one (70\%) of the 30 genotypically different $A$. baumannii isolates included in the study were isolated from tracheal aspirates, one (3.3\%) from blood, four (13.3\%) from the catheter, one (3.3\%) from a wound infection, two (6.6\%) from urine samples, and one (3.3\%) from peritoneal fluid samples. All antimicrobial susceptibility results of A. baumannii isolates are shown in Table 1 . The susceptibility results of 27 carbapenem resistant isolate (including the one with intermediate susceptibility) to antibiotics other than carbapenem are shown in Table 2 . All isolates were determined as colistin sensitive. The sensitivity rates for netilmicin, tigecycline, sulbactam, amikacin, and meropenem were $66.6 \%, 50 \%$, $36.6 \%, 30 \%$ and $10 \%$, respectively. The rates determined for carbapenem resistant isolates were $66.6 \%, 51.8 \%, 33.3 \%$, and $25.9 \%$ for netilmicin, tigecycline, sulbactam, and amikacin, respectively. The MIC50 values for netilmicin, tigecycline, sulbactam, amikacin, meropenem and colistin were $4 \mu \mathrm{g} / \mathrm{mL}, 3 \mu \mathrm{g} / \mathrm{mL}, 8 \mu \mathrm{g} / \mathrm{mL}, 128 \mu \mathrm{g} / \mathrm{mL}, 64 \mu \mathrm{g} / \mathrm{mL}$, and $1 \mu \mathrm{g} / \mathrm{mL}$, respectively, while the MIC90 values were $512 \mu \mathrm{g} / \mathrm{mL}, 8 \mu \mathrm{g} / \mathrm{mL}, 12 \mu \mathrm{g} / \mathrm{mL}, 1024 \mu \mathrm{g} / \mathrm{mL}, 128 \mu \mathrm{g} / \mathrm{mL}$, and 1 $\mu \mathrm{g} / \mathrm{mL}$, respectively.

Table 1. The Antimicrobial Susceptibility Results of A. baumannii Isolates

\begin{tabular}{lccc}
\hline & $\begin{array}{c}\text { Sensitive(S) } \\
\text { No.(\%) }\end{array}$ & $\begin{array}{c}\text { Intermediate (I) } \\
\text { No. (\%) }\end{array}$ & $\begin{array}{c}\text { Resistant(R) } \\
\text { No.(\%) }\end{array}$ \\
\hline Colistin & $30(100)$ & $0(0)$ & $0(0)$ \\
Netilmicin & $20(66.6)$ & $2(6.6)$ & $8(26.6)$ \\
Tigecycline & $15(50)$ & $13(43.3)$ & $2(6.6)$ \\
Sulbactam & $11(36.6)$ & $11(36.6)$ & $8(26.6)$ \\
Amikacin & $9(30)$ & $3(10)$ & $18(60)$ \\
Meropenem & $3(10)$ & $1(3.3)$ & $26(86.6)$ \\
\hline
\end{tabular}

Table 2. The Susceptibility Results of 27 Carbapenem Resistant Isolates to Antibiotics Other Than Meropenem

\begin{tabular}{lccc}
\hline & $\begin{array}{c}\text { Sensitive (S) } \\
\text { No.(\%) }\end{array}$ & $\begin{array}{c}\text { Intermediate (I) } \\
\text { No. (\%) }\end{array}$ & $\begin{array}{c}\text { Resistant(R) } \\
\text { No. (\%) }\end{array}$ \\
\hline Colistin & $27(100)$ & $0(0)$ & $0(0)$ \\
Netilmicin & $18(66.6)$ & $2(7.4)$ & $8(25.9)$ \\
Tigecycline & $14(51.8)$ & $11(40.7)$ & $2(7.4)$ \\
Sulbactam & $9(33.3)$ & $10(37)$ & $8(29.6)$ \\
Amikacin & $7(25.9)$ & $2(7.4)$ & $18(66.6)$ \\
\hline
\end{tabular}




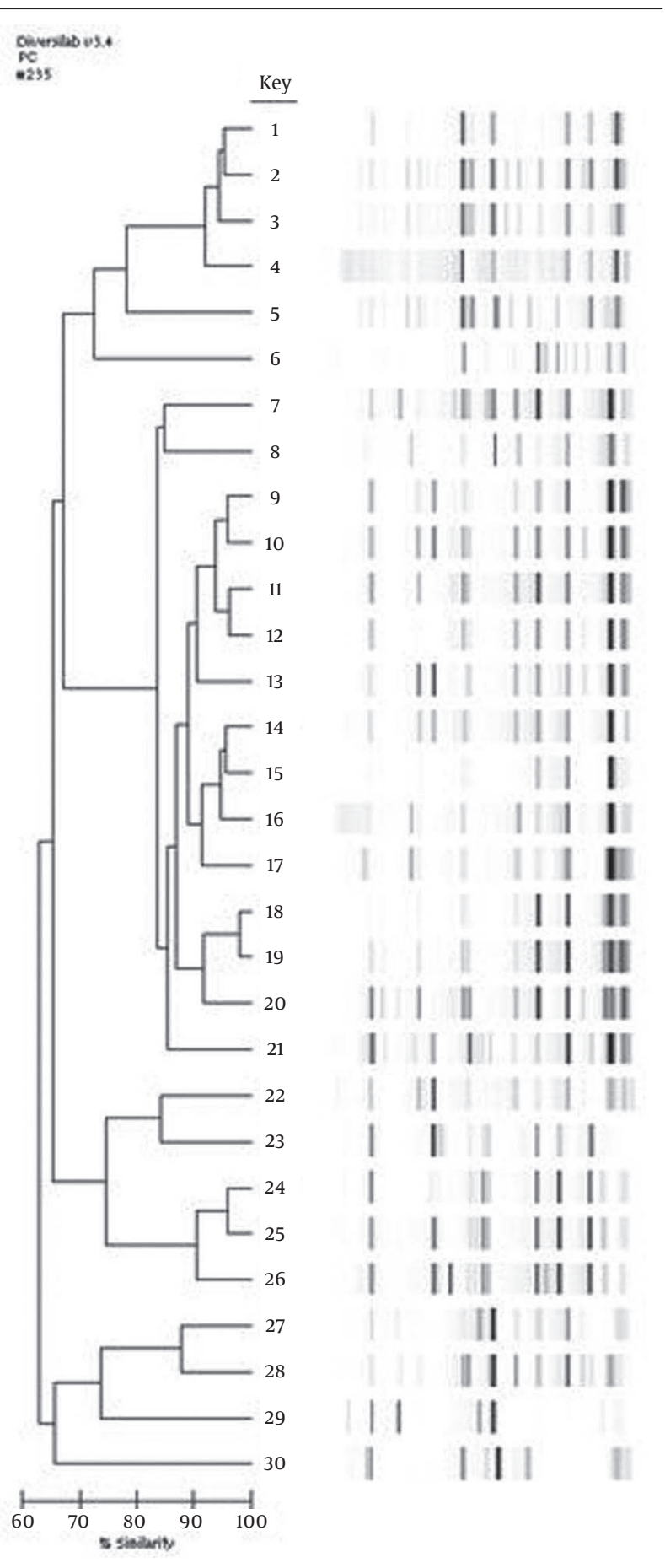

Figure 1. Genotyping Results of 300 A. baumannii Isolates

\section{Discussion}

A. baumannii has become an important health problem due to the fact that it is a pathogen that causes hospital acquired epidemics and due to treatment failures caused by multiple antibiotic resistances $(2,10)$. The same bacteria obtained from different patients in a clinic shows that there is cross contamination between the patients origi- nating from the same source. In such a case, the source of the microorganism causing the hospital acquired infection must be investigated. Through determination of the clonal relationship between the isolates, the source of the infection, the porter, and the way of spread can be revealed, and proper prevention methods can be chosen. The aim of genotyping studies is to determine whether there is an epidemiological relationship between isolated and genotyped strains and to grade this relationship if one exists. Rep-PCR is performed with primers specific to a repeated sequence in the genome and can be used in phylogenetic relationship studies for providing reliable results and repeatability (11). In a study by Caretto et al., genotyping of Acinetobacter isolates was carried out and they emphasized that rep-PCR is an expensive but a rapidly performed and repeatable method (12). Therefore in this study rep-PCR method (Diversilab, BioMérieux, France) was used for genotyping the isolates as it is a rapid and easy to use method. Only genotypically different isolates were evaluated for antimicrobial susceptibilities as the genotypically related isolates could give the same susceptibility patterns. Therefore, in the current study the antimicrobial susceptibilities were evaluated for only 30 isolates that were genotypically different and not for the 300 isolates that were collected in a two year period.

Monotherapy with sulbactam is not recommended for severe Acinetobacter infections. However, Wood et al., reported that the use of sulbactam for the treatment of 14 patients with ventilator associated pneumonia caused by multi drug resistant Acinetobacter was successful and that there was no difference between 63 patients treated with sulbactam or imipenem in terms of clinical results (13). In the study of Rodriguez-Hernandez et al., which was carried out on 150 A. baumannii isolates, it was indicated that sulbactam could be a good treatment option for multi resistant A. baumannii infections (6). They tested ampicillin, piperacillin, ticarcillin, and beta lactamase inhibitors, such as clavulanic acid, sulbactam, and tazobactam, alone or in combination. It was emphasized that beta lactamase inhibitors, with the exception of sulbactam, had no effect and ampicillin sulbactam was the most effective combination.

Corbella et al. applied intravenous treatment of sulbactam to 18 patients and intravenous treatment of ampicillin sulbactam to 24 patients out of 42 patients in which multidrug-resistant Acinetobacter was isolated. They found that the effect of ampicillin-sulbactam combination is deriven by sulbactam and this combination does not produce a synergistic effect (14). Furthermore, in an experimental model of pneumonia in which sulbactam sensitive A. baumannii was used, sulbactam was found as effective as imipenem $(6,15)$. In the current study, we found sulbactam susceptibility in a lower ratio, discordant from the literature. The sensitivity ratio of sulbactam in vitro was found to be $36.6 \%$ in all Acinetobacter isolates and $34.6 \%$ in carbapenem resistant isolates. This ratio was determined when the MIC value of sulbactam 
was accepted as sensitive if it was $\leq 4 \mu \mathrm{g} / \mathrm{mL}$. If the MIC value $\leq 8 \mu \mathrm{g} / \mathrm{mL}$ was accepted as sensitive, the sensitivity ratio would be $73.3 \%$. Furthermore, the low sulbactam susceptibility of Acinetobacter isolates in the current study may be due to selecting clones different from each other and that the isolates were multidrug resistant.

Although the carbapenems are the most effective antibiotics for the Acinetobacter species, recently in studies from Turkey and worldwide, it was reported that Acinetobacter isolates resistant to carbepenems were isolated with an increasing frequency $(2,16-21)$. There are studies from Turkey demonstrating that there is an increasing resistance to imipenem (50-84\%) and meropenem (63$80.3 \%$ ) (18-21). In the current study, there was $86.6 \%$ resistance to meropenem and $3.3 \%$ of the isolates were intermediate. The high levels of carbapenem resistance may be due to the multidrug resistance of the isolates and the prolonged duration of empirical treatment with carbapenem at our studied hospital.

This study is the first study from Turkey where the MIC value of sulbactam in Acinetobacter was determined alone in vitro. Further clinical studies are needed to determine the efficacy of sulbactam on A. baumannii. In the current study, the MIC value of sulbactam $\leq 4 \mu \mathrm{g} / \mathrm{mL}$ was accepted as susceptible, the value of $8 \mu \mathrm{g} / \mathrm{mL}$ was accepted as intermediate and the value of $\geq 16 \mu \mathrm{g} / \mathrm{mL}$ was accepted as resistant. If we had determine the cut-off MIC value two folds higher it would have considerably affected the susceptibility results. The number of carbapenem resistant Acinetobacter species is increasing gradually. Therefore, it is important to determine the resistance profile for hospital settings in order to choose the proper antibiotic for empirical treatment.

\section{References}

1. Larsen AR. Nosocomial infections. In: Hoeprich PD, Jordan MC editors. Infectious Disease. 4 ed. Phledelpiha: J.B.Lippincott Company; 1989. pp. 35-40.

2. Ferrara AM. Potentially multidrug-resistant non-fermentative Gram-negative pathogens causing nosocomial pneumonia. Int J Antimicrob Agents. 2006;27(3):183-95.

3. Akalın, H.. Çoğul Dirençli Gram Negatif Bakteriler. In: Doğanay M, Ünal S editors. Hastane infeksiyonlan.. Ankara: Bilimsel Tıp Yayınevi; 2003. pp. 269-87.

4. Higgins PG, Wisplinghoff $H$, Stefanik D, Seifert $H$. In vitro activities of the beta-lactamase inhibitors clavulanic acid, sulbactam, and tazobactam alone or in combination with beta-lactams against epidemiologically characterized multidrug-resistant Acinetobacter baumannii strains. Antimicrob Agents Chemother. 2004;48(5):1586-92.

5. Bello H, Dominguez M, Gonzalez G, Zemelman R, Mella S, Young $\mathrm{HK}$, et al. In vitro activities of ampicillin, sulbactam and a combination of ampicillin and sulbactam against isolates of Aci- netobacter calcoaceticus-Acinetobacter baumannii complex isolated in Chile between 1990 and 1998. J Antimicrob Chemother. 2000;45(5):712-3.

6. Rodriguez-Hernandez MJ, Cuberos L, Pichardo C, Caballero FJ, Moreno I, Jimenez-Mejias ME, et al. Sulbactam efficacy in experimental models caused by susceptible and intermediate Acinetobacter baumannii strains. J Antimicrob Chemother 2001;47(4):479-82.

7. Fontana C, Favaro M, Minelli S, Bossa MC, Testore GP, Leonardis F, et al. Acinetobacter baumannii in intensive care unit: a novel system to study clonal relationship among the isolates. BMC Infect Dis. 2008;8:79.

8. Navon-Venezia S, Leavitt A, Carmeli Y. High tigecycline resistance in multidrug-resistant Acinetobacter baumannii. J Antimicrob Chemother. 2007;59(4):772-4.

9. Clinical and Laboratory Standards Institute . Performance standards for antimicrobial disk susceptibility tests. Approved Standard M2-A9. 9 edWayne, Pennsylvania: CLSI; 2010.

10. Chuang YC, Sheng WH, Lauderdale TL, Li SY, Wang JT, Chen YC et al. Molecular epidemiology, antimicrobial susceptibility and carbapenemase resistance determinants among Acinetobacter baumannii clinical isolates in Taiwan. J Microbiol Immunol Infect. 2014;47(4):324-32.

11. Olive DM, Bean P. Principles and applications of methods for DNA-based typing of microbial organisms. J Clin Microbiol. 1999;37(6):1661-9.

12. Carretto E, Barbarini D, Farina C, Grosini A, Nicoletti P, Manso E, et al. Use of the DiversiLab semiautomated repetitive-sequencebased polymerase chain reaction for epidemiologic analysis on Acinetobacter baumannii isolates in different Italian hospitals. Diagn Microbiol Infect Dis. 2008;60(1):1-7.

13. Wood GC, Hanes SD, Croce MA, Fabian TC, Boucher BA. Compari son of ampicillin-sulbactam and imipenem-cilastatin for the treatment of acinetobacter ventilator-associated pneumonia. Clin Infect Dis. 2002;34(11):1425-30.

14. Corbella X, Ariza J, Ardanuy C, Vuelta M, Tubau F, Sora M, et al. Efficacy of sulbactam alone and in combination with ampicillin in nosocomial infections caused by multiresistant Acinetobacter baumannii. JAntimicrob Chemother. 1998;42(6):793-802.

15. Joly-Guillou ML, Wolff M, Farinotti R, Bryskier A, Carbon C. In vivo activity of levofloxacin alone or in combination with imipenem or amikacin in a mouse model of Acinetobacter baumannii pneumonia. JAntimicrob Chemother. 2000;46(5):827-30.

16. Ruiz J, Nunez ML, Perez J, Simarro E, Martinez-Campos L, Gomez J. Evolution of resistance among clinical isolates of Acinetobacter over a 6-year period. Eur J Clin Microbiol Infect Dis. 1999;18(4):2925.

17. Jeong SH, Bae IK, Park KO, An YJ, Sohn SG, Jang SJ, et al. Outbreaks of imipenem-resistant Acinetobacter baumannii producing carbapenemases in Korea.J Microbiol. 2006;44(4):423-31.

18. Yavuz MT, Şahin, İ. , Behçet, M. , Öztürk, E. , Kaya, D. . Antibiotic Susceptibility of Acinetobacter baumannii Strains Isolated from Various Clinical Samples. ANKEM. 2006;20(2):107-710.

19. Ozdem B, Gurelik FC, Celikbilek N, Balikci H, Acikgoz ZC. [Antibiotic resistance profiles of Acinetobacter species isolated from several clinical samples between 2007-2010]. Mikrobiyol Bul. 2011;45(3):526-34.

20. Turk Dagi H, Arslan U, Tuncer I. Antibiotic Resistance of Acinetobacter baumannii Strains Isolated from Blood Cultures. ANKEM Dergisi. 2011;25(1):27-30.

21. Alp E, Esel D, Yildiz O, Voss A, Melchers W, Doganay M. Genotypic analysis of Acinetobacter bloodstream infection isolates in a Turkish university hospital. Scand J Infect Dis. 2006;38(5):335-40. 